\title{
Laser Treatments for Vitiligo
}

\author{
Jung Min Bae ${ }^{1}$ \\ Seung-Kyung Hann²
}

${ }^{1}$ Department of Dermatology, St. Vincent's
Hospital, College of Medicine, The Catholic
University of Korea, Suwon, Korea
${ }^{2}$ Korea Institute of Vitiligo Research and Drs Woo
and Hann's Skin Center, Seoul, Korea

Received November 20, 2016

Accepted November 24, 2016

\section{Correspondence}

Jung Min Bae

Department of Dermatology, St. Vincent's Hospital, College of Medicine, The Catholic University of Korea, 93 Jungbu-daero, Paldal-gu, Suwon 16247, Korea

Tel.: +82-31-249-7461

Fax: +82-31-242-8927

E-mail: jminbaedgmail.com

(C) Korean Society for Laser Medicine and Surgery

(c) This is an open access article distributed under the terms of the Creative Commons Attribution NonCommercial License (http://creativecommons.org/ licenses/by-nc/4.0) which permits unrestricted noncommercial use, distribution, and reproduction in any medium, provided the original work is properly cited.
Vitiligo remains to be a major challenge in the field of dermatology. To this day, there is still no definitive cure. Although phototherapy has been the mainstay to manage vitiligo, it is associated with several adverse effects, such as tanning, burning, and skin aging, due to the unavoidable exposure of normal, uninvolved skin to intense ultraviolet radiation. Laser treatment has an advantage in treating vitiligo in a targeted way, and it appears to be a reasonable option for localized vitiligo. Moreover, a majority of patients have limited involvement of body surface area. An increasing number of reports have highlighted the value of lasers as a treatment option for vitiligo. The 308-nm xenon chloride excimer laser is already considered to be a treatment of choice for localized vitiligo. Additionally, there are emerging lasers that have been attempted as the treatment of vitiligo, including low-energy 632.8-nm helium-neon lasers, ablative fractional lasers, gain-switched 311-nm titanium:sapphire laser, and 355-nm monochromatic ultraviolet A1 laser. While evidences for these laser treatments of vitiligo are limited, some results seem to be promising. For example, studies have shown a responsiveness in otherwise refractory cases and via different mechanisms of action. However, most studies were uncontrolled and non-comparative, and have produced no conclusive results. Nonetheless, it is expected that we will witness greater development and advancement in laser treatments for vitiligo soon. In this article, we discuss the current state of laser treatments for vitiligo and proposed mechanisms of action.

\section{Key words}

Excimer; Laser treatment; Vitiligo 


\section{INTRODUCTION}

Vitiligo is a common acquired depigmentation disorder, resulting from the selective loss of melanocytes in the skin and mucosa. 'It affects $0.5-2 \%$ of the population and remains a major challenge in dermatology because there is no definitive cure. Although it is generally asymptomatic, patients with vitiligo suffer from profound psychological distress, especially in ethnic populations. ${ }^{2}$ Phototherapy, including psoralen plus ultraviolet $A$ and narrow-band ultraviolet B (NBUVB) therapy, has been the mainstay for vitiligo. ${ }^{3}$ However, phototherapy is associated with several adverse effects, including tanning, burning, and skin aging, when administered over the long term, because normal, uninvolved skin is unavoidably exposed to intense ultraviolet radiation. ${ }^{4}$
An increasing number of reports have highlighted the value of lasers in the treatment of vitiligo (Table 1). The $308-\mathrm{nm}$ xenon chloride excimer laser is the most widely used laser for vitiligo, and is currently considered the treatment of choice for localized vitiligo. ${ }^{5}$ Additionally, there are several other emerging lasers in the treatment of vitiligo, including low-energy 632.8-nm helium-neon (He-Ne) lasers, ablative fractional lasers, a gain-switched 311-nm titanium:sapphire (Ti:Sapphire) laser, and a 355nm monochromatic ultraviolet A1 (UVA1) laser. However, most of them have demonstrated effectiveness only in small numbers of pilot studies.

Lasers have an advantage in treating vitiligo; they allow selective treatment of depigmented lesions, preventing unnecessary irradiation of uninvolved skin. ${ }^{5}$ Thus, laser treatment appears to be a reasonable option for localized

Table 1. Laser treatments for vitiligo

\begin{tabular}{|c|c|c|}
\hline Laser modality & Clinical response & Mechanism of action \\
\hline $\begin{array}{l}\text { 308-nm xenon chloride } \\
\text { excimer laser }\end{array}$ & $\begin{array}{l}\text { The 308-nm excimer laser is the most validated laser modality } \\
\text { in vitiligo treatment. } \\
\text { Treatment success (> } 75 \% \text { repigmentation) was generally } \\
\text { achieved in } 15-61 \% \text { of each vitiliginous patch. } \\
\text { The most effective responses would be anticipated on the } \\
\text { face and neck, while the hands and feet show minimal } \\
\text { responses. }\end{array}$ & $\begin{array}{l}\text { It can contribute to both immunomodulation and the migration } \\
\text { and proliferation of melanocytes, like NBUVB. } \\
\text { The excimer laser has been found to be a more potent inducer } \\
\text { of T cell apoptosis than NBUVB. } \\
\text { Both the excimer laser and NBUVB can increase the level of } \\
\text { endothelin-1 mRNA at a dose close to the minimal erythema } \\
\text { dose. } \\
\text { The excimer laser induced immature pigment cell develop- } \\
\text { ment that was not seen with NBUVB treatment }\end{array}$ \\
\hline $\begin{array}{l}\text { Low-energy 632.8-nm } \\
\text { He-Ne laser }\end{array}$ & $\begin{array}{l}\text { He-Ne laser treatment was effective in patients with seg- } \\
\text { mental vitiligo on the head/neck in a pilot study. Marked } \\
\text { responses (> 50\% repigmentation) were achieved in } 60 \% \text { of } \\
\text { patients after a median of } 118 \text { treatment sessions. }\end{array}$ & $\begin{array}{l}\text { Medium from He-Ne laser-irradiated keratinocytes enhanced } \\
\text { melanocyte migration, possibly by increasing basic fibro- } \\
\text { blast growth factor and nerve growth factor. } \\
\text { It could normalize dysfunction in cutaneous blood flow and } \\
\text { adrenoceptor responses in segmental vitiligo. } \\
\text { It stimulated primitive pigment cell differentiation, an effect } \\
\text { not seen with UVB treatment. }\end{array}$ \\
\hline Ablative fractional laser & $\begin{array}{l}\text { An additive effect of ablative fractional CO2 laser (100 mJ/ } \\
\text { spot) with NBUVB phototherapy for vitiligo has been shown. } \\
\text { Combined treatment with a fractional CO2 laser plus high- } \\
\text { potency topical corticosteroids and NBUVB showed an } \\
\text { effective treatment response in refractory vitiligo. } \\
\text { The addition of low-fluence fractional CO2 laser treatment } \\
\text { (4 mJ/spot) showed minimal repigmentation in } 21.7 \% \text { of } \\
\text { patients with refractory vitiligo. }\end{array}$ & $\begin{array}{l}\text { Various cytokines and growth factors that are secreted during } \\
\text { wound healing may act as mitogens for melanocytes. } \\
\text { A significant increase in endothelin-1 mRNA expression was } \\
\text { observed after low-fluence fractional CO2 laser treatment at } \\
2 \mathrm{~mJ} / \mathrm{spot} \text {. }\end{array}$ \\
\hline $\begin{array}{l}\text { Gain-switched 311-nm } \\
\text { T1:Sapphire laser }\end{array}$ & $\begin{array}{l}\text { A TI:Sapphire laser was shown to be as effective as an } \\
\text { excimer laser. } \\
\text { The minimal erythema dose of a Ti:Sapphire laser is } 1.7 \text { times } \\
\text { that of 308-nm excimer lasers. }\end{array}$ & $\begin{array}{l}\text { Immunosuppressive effects of the Ti:Sapphire laser have } \\
\text { been observed in atopic dermatitis animal models. } \\
\text { Increased endothelin-1 mRNA, a melanogenic paracrine } \\
\text { cytokine, was also observed. }\end{array}$ \\
\hline $\begin{array}{c}\text { 355-nm monochromatic } \\
\text { ultraviolet A1 laser }\end{array}$ & $\begin{array}{l}\text { Clinical repigmentation was observed in } 15 / 17 \text { ( } 88.2 \%) \\
\text { patients, and } 52.9 \% \text { (9/17) of patients showed good-to- } \\
\text { excellent responses (> 50\% repigmentation). }\end{array}$ & $\begin{array}{l}\text { UVA1 wavelengths penetrate the skin more deeply than UVB, } \\
\text { thus effectively better modulating the activities of immune } \\
\text { and inflammatory cell populations in the dermis. } \\
\text { UVA1 phototherapy modulated pro inflammatory cytokines, } \\
\text { including tumor necrosis factor- } \alpha \text {, interleukin-12, and } \\
\text { interferon- } \gamma \text {. } \\
\text { It also stimulated melanocyte activity. }\end{array}$ \\
\hline
\end{tabular}


vitiligo. Furthermore, children would be another indication for laser treatment because it does not require entering a phototherapy unit that could limit pediatric use. Although evidence for the use of lasers in the treatment of vitiligo is currently limited, some results are promising. In this article, we discuss the current state of laser treatments for vitiligo and proposed mechanisms of action.

\section{THE 308-NM EXCIMER LASER}

The 308-nm xenon chloride excimer laser was first described for the treatment of vitiligo in 2001, ${ }^{6}$ and the US Food and Drug Administration approved it for the treatment of vitiligo in 2007. ' It emits a monochromatic wavelength of $308 \mathrm{~nm}$ and induces photobiological effects similar to those of NBUVB that have maximum emission at $311 \mathrm{~nm}$. It also delivers a higher fluence to depigmented lesions selectively, sparing uninvolved normal skin. There are only a few products available on the market, including XTRAC (PhotoMedex Inc., Horsham Township, PA, USA) and E1 (Pronexx Co. Ltd., Seoul, Korea).

\section{Efficacy of the excimer laser}

Several studies have assessed the efficacy of 308$\mathrm{nm}$ excimer lasers in the treatment of vitiligo. There is a great diversity in treatment responses among studies, and treatment success ( $>75 \%$ repigmentation) is generally anticipated in between 15 and $61 \%$ of each vitiliginous patch. ${ }^{8-14}$ However, the treatment response is dependent largely on body site. ${ }^{9,11,12}$ In a recent retrospective study of 979 patients by Fa et al., ${ }^{12}$ the best response was noted on the face ( $>75 \%$ repigmentation: $40.86 \%$ ) whereas the hands and feet showed the poorest response $1>75 \%$ repigmentation: $9.17 \%$ ).

\section{Comparison of the excimer laser and NBUVB}

There is currently little evidence to support the superior efficacy of excimer lasers compared with NBUVB. Hong et al..$^{10}$ first compared the clinical efficacy of a 308$\mathrm{nm}$ excimer laser with that of NBUVB in a pilot study of 23 vitiliginous patches in eight patients. They showed both better effectiveness and earlier appearance of repigmentation with 308-nm excimer laser treatment than with NBUVB treatment. However, Linthorst Homan et al. ${ }^{15}$ conducted a randomized comparison of an excimer laser versus NBUVB phototherapy after punch grafting in 14 patients with stable vitiligo, and showed no significant difference in the grade of repigmentation at 3 months. Regarding 308-nm monochromatic excimer light treatment, the superiority of excimer light over NBUVB is also controversial with conflicting results among different studies. ${ }^{16-18}$

Meanwhile, the excimer laser has shown to be associated with a faster onset of repigmentation and fewer treatment sessions for a successful response compared with conventional phototherapy. Zhang et al..$^{13}$ observed the onset of repigmentation after an average of 6.1 treatment sessions with an excimer laser, and Xiang et al. ${ }^{14}$ showed that $45.7 \%$ (92/201) of all patches treated with an excimer laser showed signs of repigmentation within the first 4 treatment sessions.

\section{Treatment protocol}

The treatment protocol for an excimer laser varies according to the clinical setting. Treatment is generally started at $100-300 \mathrm{~mJ} / \mathrm{cm}^{2}$, depending on the body site and Fitzpatrick skin type. The dose is increased by $50 \mathrm{~mJ} /$ $\mathrm{cm}^{2}$ at each session until post-treatment erythema occurs, and the dose is then kept constant at the minimal erythema dose. A dose that will induce asymptomatic erythema, present for 24 to 48 hours after treatment, is considered ideal. Treatment sessions can be conducted one, two, or three times weekly. However, the ultimate rate of repigmentation seems to depend on the total number of treatment sessions, not their frequency, although repigmentation occurs more rapidly with more frequent treatment. ${ }^{19}$ Treatment is commonly performed on two non-consecutive days per week. We suggest a treatment protocol of the 308-nm excimer laser for vitiligo in Table 2 . based on our experience and published ones. ${ }^{10,20}$

\section{Mechanism of action}

Although the exact mechanism of action of an excimer laser in vitiligo is not fully understood, ultraviolet B (UVB) light seems to contribute to both modulating the immune response and the migration and proliferation of melanocytes. ${ }^{4}$ In early studies of psoriasis, a 308-nm excimer laser was shown to be more effective at inducing $T$ cell apoptosis in lesional skin than NBUVB, ${ }^{21,22}$ and this immunomodulatory effect could also be responsible for the treatment response in vitiligo. During phototherapy, repigmentation begins at the hair follicle in many cases, and both NBUVB and excimer laser treatment have been shown to potentially induce melanocyte migration and proliferation from the niche located in hair follicles. ${ }^{23-25}$ Various cytokines, including stem cell factor, $\alpha$-melanocyte-stimulating hormone, and endothelin-1, have been shown to be associated with mitogenesis and the migration of melanocytes during phototherapy, ${ }^{26,27}$ and an excimer laser dose close to the minimal erythema dose was 
Table 2. Treatment protocol of the 308-nm excimer laser for vitiligo

\begin{tabular}{lc}
\hline \multicolumn{1}{c}{ Body site } & Initial dose \\
\hline Face and neck & $150 \mathrm{~mJ} / \mathrm{cm}^{2}$ \\
Trunk and extremities & $200 \mathrm{~mJ} / \mathrm{cm}^{2}$ \\
Hands and feet & $300 \mathrm{~mJ} / \mathrm{cm}^{2}$ \\
\hline \multicolumn{1}{c}{ Post-treatment response } & Dose modification \\
\hline No erythema & Increase dose by $50 \mathrm{~mJ} / \mathrm{cm}^{2}$ \\
Transient asymptomatic erythema & Maintain dose \\
within 48 hours & Decrease dose by $50 \mathrm{~mJ} / \mathrm{cm}^{2}$ \\
Persistent asymptomatic erythema & then reduce dose by $100 \mathrm{~mJ} / \mathrm{cm}^{2}$ \\
over 48 hours & General treatment principle \\
Persistent symptomatic erythema & Hold treatment until resolved, \\
with pain or blister & \\
\hline & \\
\hline A dose that induces asymptomatic erythema for $24-48$ hours after treat- \\
ment is ideal. \\
Treatment should be performed on two non-consecutive days per week. \\
Sunscreens should be removed from the treatment area because they \\
absorb UVB light. \\
For pediatric use, a dose reduction of $50 \mathrm{~mJ} / \mathrm{cm}^{2}$ from the initial dose is \\
recommended.
\end{tabular}

demonstrated to potentially increase the level of endothelin-1 mRNA. ${ }^{28}$ Recently, Lan et al. ${ }^{29}$ demonstrated that irradiance $\left(\mathrm{mW} / \mathrm{cm}^{2}\right)$, rather than fluence $\left(\mathrm{mJ} / \mathrm{cm}^{2}\right)$, played an important role in UVB-induced immature pigment cell development; these results support the possible superiority of an excimer laser over NBUVB in primitive melanoblasts.

\section{Adverse effects}

Post-treatment erythema is observed frequently, while burning, blisters, and itching are seen occasionally. ${ }^{7}$ Choi et al. ${ }^{9}$ reported that the most common adverse effects were long-lasting or symptomatic erythema (48.5\%), while perilesional hyperpigmentation (14.3\%) and blister formation (10.7\%) were minimal, in their retrospective review of 69 patients. Most adverse effects were generally tolerable, and no serious adverse effect was reported.

\section{LOW-ENERGY 632.8-NM HELIUM-NEON LASER}

A low-energy 632.8-nm He-Ne laser (Omniprobe Laser Biostimulation System; Physio Technology, Topeka, KS, USA) has been addressed in the treatment of segmental vitiligo by a Taiwanese group. ${ }^{30}$ Recently, a successful He-Ne laser (HLA-2000 Laser Therapy System; Hanil Meditech, Seoul, Koreal and topical tacrolimus combination therapy in one child with non-segmental vitiligo was reported in Korea. ${ }^{31}$

\section{Clinical studies}

Yu et al. ${ }^{30}$ performed He-Ne laser treatment in 30 patients with segmental vitiligo on the head and neck. The laser was administered locally at a fixed fluence of 3.0 $\mathrm{J} / \mathrm{cm}^{2}$ once or twice weekly. Initial repigmentation was observed after an average of 16 treatment sessions, and marked repigmentation ( $>50 \%$ ) was achieved in $60 \%$ of patients after a median of 118 treatment sessions (range, $16-148)$. In 3 of $30(10 \%)$ patients, complete repigmentation occurred after 16,20 , and 24 treatment sessions, respectively. Only $16.7 \%$ (5/30) showed a poor response $(<25 \%$ ) after a median of 50 treatment sessions (range, $37-100)^{30}$

\section{Mechanism of action}

An in vitro study showed that significant increases in basic fibroblast growth factor and nerve growth factor released from keratinocytes after He-Ne laser treatment, and the medium from He-Ne laser-irradiated keratinocytes could directly enhance melanocyte migration. ${ }^{30}$ The same group also showed that He-Ne laser treatment could normalize cutaneous blood flow, which was significantly higher in segmental vitiligo compared with contralateral normal skin, and they concluded that He-Ne laser therapy could normalize dysfunction in cutaneous blood flow and adrenoceptor responses in patients with segmental vitiligo. ${ }^{32}$ They also showed that $\mathrm{He}-\mathrm{Ne}$ laser irradiation promoted melanoblast differentiation via mitochondrial retrograde signaling, an effect not replicable with UVB treatment, even at high and damaging doses. ${ }^{33}$

\section{Limitations}

Despite interesting results from laboratory and clinical studies of patients with segmental vitiligo, additional trials are needed to confirm the indications for the use of HeNe lasers in vitiligo.

\section{ABLATIVE FRACTIONAL LASER}

The ablative fractional laser was first developed for the remodeling of scars; it has also been tried to induce repigmentation in vitiligo. There have been a series of reports of the clinical improvement in vitiligo after dermabrasion ${ }^{34,35}$ and ablative laser treatments..$^{36,37}$ The ablative fractional laser does not ablate the entire epidermis, leaving normal intact skin between the coagulated necrotic columns. It decreases the risk of potential side effects, and re-epithelialization occurs within 24 hours via keratinocyte migration from the surrounding normal tissue. $^{38}$ 


\section{Clinical studies}

Shin et al..$^{39}$ first showed the additive effect of a 10,600$\mathrm{nm}$ ablative fractional $\mathrm{CO}_{2}$ laser leCO2; Lutronic Corp. Goyang, South Koreal to conventional NBUVB phototherapy for refractory vitiligo in their prospective, randomized, left-right comparative study of ten patients. All patients had symmetrical vitiligo lesions without improvement despite more than 1 year of conventional treatment. The laser was administrated with 2 passes of a pulse energy of $100 \mathrm{~mJ} / \mathrm{spot}$ and a spot density of $150 \mathrm{spots} / \mathrm{cm}^{2}$ at a 2-month interval. At 2 months after the 2 courses of ablative fractional treatment, $30 \%$ of patients showed more than a moderate response ( $>25 \%$ repigmentation) to combined treatment, while none reached more than a moderate response with NBUVB phototherapy alone. Thereafter, triple combination treatment with a fractional $\mathrm{CO}_{2}$ laser, high potency topical corticosteroids, and NBUVB also produced an effective treatment response in refractory vitiligo in two recent prospective, randomized split-body, comparative studies. , $^{4,41}$

Recently, we performed a retrospective study of the addition of low-fluence fractional $\mathrm{CO}_{2}$ laser treatment for refractory vitiligo not responsive to 308-nm excimer laser treatment over 6 months. In total, 46 patients were enrolled, and the laser was used weekly with two passes at a pulse energy of $4 \mathrm{~mJ} / \mathrm{spot}$ and a spot density of 300 spots $/ \mathrm{cm}^{2}$. Repigmentation was evaluated after five treatment sessions, and $21.7 \%$ (10/46) of the patients showed at least minimal repigmentation (in press).

\section{Mechanism of action}

It has been suggested that dermabrasion enhances the repigmentation rate by increasing penetration of the dermis by ultraviolet radiation, removing affected keratinocytes, and inducing various kinds of cytokines and growth factors that may act as mitogens for melanocytes. ${ }^{36,42}$ Theoretically, an ablative fractional laser would be expected to share the same mechanisms as dermabrasion and ablative lasers, but would minimize the side effects. ${ }^{39}$ In our in vivo study, a significant increase in endothelin-1 mRNA expression was observed at 24 hours after lowfluence fractional $\mathrm{CO}_{2}$ laser treatment at 2 and $4 \mathrm{~mJ} /$ spot (in press). In addition, microscopic ablative zones produced with a fractional $\mathrm{CO}_{2}$ laser would be expected to promote the transepidermal penetration of topical agents, which may provide additional benefits for repigmentation in vitiligo. ${ }^{40,41}$

\section{Limitations}

In most studies, fractional laser treatment was applied as an adjuvant treatment to conventional phototherapy. Clinical trials to evaluate the genuine efficacy of fractional laser treatment alone are required to justify the use of the laser in the treatment of vitiligo.

\section{GAIN-SWITCHED 311-NM TITANIUM:SAPPHIRE LASER}

A gain-switched 311-nm Ti:Sapphire laser (Pallas; Laseroptek, Seongnam, Koreal has been developed following the peak spectrum of NBUVB (311 nm). It was approved by the Korean Food and Drug Administration for the treatment of psoriasis and vitiligo in 2015. The 311-nm Ti:Sapphire laser has some advantages compared with the 308-nm excimer laser, including deeper penetration by the $311 \mathrm{~nm}$ wavelength and the use of a solid medium with reduced maintenance costs.

\section{Clinical studies}

We performed a randomized, controlled, non-inferiority trial, based on a split-body protocol for the treatment of vitiligo. ${ }^{43}$ The minimal erythema dose of the $311-n m$ Ti:Sapphire laser was 1.7 times that of the 308-nm excimer laser in a pretest of ten volunteers. A total of 52 paired lesions in 16 patients were assigned to either the excimer laser treatment group or the Ti:Sapphire laser treatment group so far. Twenty-two paired lesions completed the 12-week trial course, and an interim analysis showed that the Ti:Sapphire laser is as effective as the excimer laser in the treatment of vitiligo. ${ }^{43}$ The profiles of adverse effects of the two lasers were similar; no serious adverse effect was observed in either group.

\section{Mechanism of action}

Ti:Sapphire laser treatment seems to be associated with immune modulation and melanocyte stimulation, like excimer laser treatment. The laser was demonstrated to effectively suppress the immune response and provide clinical improvement in an atopic dermatitis animal model. ${ }^{44}$ Additionally, an increase in endothelin-1 mRNA, a melanogenic paracrine cytokine, was observed after Ti:Sapphire laser treatment in an in vivo animal study. ${ }^{43}$

\section{5-NM ULTRAVIOLET A1 LASER}

A 355-nm monochromatic UVA1 laser was first introduced to treat psoriasis. ${ }^{45}$ It has also been demonstrated to be effective in the treatment of vitiligo. ${ }^{46}$ 


\section{Clinical studies}

Babino et al. ${ }^{46}$ conducted an open-label, prospective study of 17 patients with vitiligo. The 355-nm UVA1 laser treatment was conducted twice weekly for 8 weeks at a fixed dose of 80-140 J/cm². Repigmentation was observed in 15 of 17 (88.2\%) patients, and 9 (52.9\%) showed good to excellent responses (>50\% repigmentation). Adverse effects, including mild post-treatment erythema and itching, were observed rarely. The major limitations of the study were the absence of a comparison group and the small number of patients.

\section{Mechanism of action}

UVA1 wavelengths penetrate the skin more deeply than UVB, possibly better modulating the activities of immune and inflammatory cell populations in the dermis. It has been demonstrated that UVA1 phototherapy can modulate proinflammatory cytokines, including tumor necrosis factor- $\alpha$, interleukin-12, and interferon- $\gamma .{ }^{47}$ Furthermore, UVA1 stimulates melanocyte activity, increasing melanin density and elongation and branching of melanocyte dendrites, resulting clinically in increased pigmentation or tanning.

\section{CONCLUSION}

Although there are several therapeutic modalities available, the treatment of vitiligo remains difficult. NBUVB phototherapy, which is used most commonly for patients with vitiligo, shows limited responses in certain patients, and is associated with unnecessary ultraviolet radiation exposure to normal skin. Moreover, a majority of patients have limited involvement of body surface area. In a recent nationwide study by the Korean Society of Vitiligo, 75.3\% of enrolled patients $(n=1,272)$ showed involvement of $<5 \%$ of the body surface area. ${ }^{48}$ Thus, laser treatment is worthy of note, and various lasers have been tried in the treatment of vitiligo over the last decade. Some studies have shown promising results, with responsiveness in otherwise refractory cases, and with differing mechanisms of action, although most of them were uncontrolled and non-comparative. We expect the further development and advances in the laser treatment of vitiligo in near future.

\section{REFERENCES}

1. Alikhan A, Felsten LM, Daly M, Petronic-Rosic V. Vitiligo: a comprehensive overview Part I. Introduction, epidemiology, quality of life, diagnosis, differential diagnosis, associations, histopathology, etiology, and work-up. J Am Acad Dermatol
2011;65:473-91.

2. Kim DY, Lee JW, Whang SH, Park YK, Hann SK, Shin YJ. Quality of life for Korean patients with vitiligo: Skindex-29 and its correlation with clinical profiles. J Dermatol 2009;36:317-22.

3. Njoo MD, Spuls PI, Bos JD, Westerhof W, Bossuyt PM. Nonsurgical repigmentation therapies in vitiligo. Meta-analysis of the literature. Arch Dermatol 1998;134:1532-40.

4. Pacifico A, Leone G. Photolchemoltherapy for vitiligo. Photodermatol Photoimmunol Photomed 2011;27:261-77.

5. Park KK, Liao W, Murase JE. A review of monochromatic excimer light in vitiligo. Br J Dermatol 2012;167:468-78.

6. Baltás E, Nagy P, Bónis B, Novák Z, Ignácz F, Szabó G, et al. Repigmentation of localized vitiligo with the xenon chloride laser. Br J Dermatol 2001;144:1266-7.

7. Sun Y, Wu Y, Xiao B, Li L, Li L, Chen HD, et al. Treatment of 308$\mathrm{nm}$ excimer laser on vitiligo: A systemic review of randomized controlled trials. J Dermatolog Treat 2015;26:347-53.

8. Nicolaidou E, Antoniou C, Stratigos A, Katsambas AD. Narrowband ultraviolet B phototherapy and 308-nm excimer laser in the treatment of vitiligo: a review. J Am Acad Dermatol 2009;60:470-7.

9. Choi KH, Park JH, Ro YS. Treatment of Vitiligo with 308-nm xenon-chloride excimer laser: therapeutic efficacy of different initial doses according to treatment areas. J Dermatol 2004;31: 284-92.

10. Hong SB, Park HH, Lee MH. Short-term effects of 308-nm xenon-chloride excimer laser and narrow-band ultraviolet $\mathrm{B}$ in the treatment of vitiligo: a comparative study. J Korean Med Sci 2005;20:273-8.

11. Hadi S, Tinio P, Al-Ghaithi K, Al-Qari H, Al-Helalat M, Lebwohl $\mathrm{M}$, et al. Treatment of vitiligo using the 308-nm excimer laser. Photomed Laser Surg 2006;24:354-7.

12. Fa Y, Lin Y, Chi XJ, Shi WH, Wang JL, Guo X, et al. Treatment of vitiligo with 308-nm excimer laser: our experience from a 2-year follow-up of 979 Chinese patients. J Eur Acad Dermatol Venereol. In press 2016.

13. Zhang $X Y, H e ~ Y L$, Dong J, Xu JZ, Wang J. Clinical efficacy of a $308 \mathrm{~nm}$ excimer laser in the treatment of vitiligo. Photodermatol Photoimmunol Photomed 2010;26:138-42

14. Xiang L. Once-weekly treatment of vitiligo with monochromatic excimer light $308 \mathrm{~nm}$ in Chinese patients. J Eur Acad Dermatol Venereol 2008;22:899-900.

15. Linthorst Homan MW, Spuls PI, Nieuweboer-Krobotova L, de Korte J, Sprangers MA, Bos JD, et al. A randomized comparison of excimer laser versus narrow-band ultraviolet $B$ phototherapy after punch grafting in stable vitiligo patients. J Eur Acad Dermatol Venereol 2012;26:690-5.

16. Verhaeghe E, Lodewick E, van Geel N, Lambert J. Intrapatient comparison of 308-nm monochromatic excimer light and localized narrow-band UVB phototherapy in the treatment 
of vitiligo: a randomized controlled trial. Dermatology 2011;223:343-8.

17. Leone G, lacovelli P, Paro Vidolin A, Picardo M. Monochromatic excimer light $308 \mathrm{~nm}$ in the treatment of vitiligo: a pilot study. J Eur Acad Dermatol Venereol 2003;17:531-7.

18. Casacci M, Thomas P, Pacifico A, Bonnevalle A, Paro Vidolin A, Leone G. Comparison between 308-nm monochromatic excimer light and narrowband UVB phototherapy (311-313 nm) in the treatment of vitiligo--a multicentre controlled study. J Eur Acad Dermatol Venereol 2007;21:956-63.

19. Hofer A, Hassan AS, Legat FJ, Kerl H, Wolf P. Optimal weekly frequency of 308-nm excimer laser treatment in vitiligo patients. Br J Dermatol 2005;152:981-5.

20. Beggs S, Short J, Rengifo-Pardo M, Ehrlich A. Applications of the excimer laser: a review. Dermatol Surg 2015;41:1201-11.

21. Novák Z, Bónis B, Baltás E, Ocsovszki I, Ignácz F, Dobozy A, et al. Xenon chloride ultraviolet $B$ laser is more effective in treating psoriasis and in inducing $T$ cell apoptosis than narrowband ultraviolet B. J Photochem Photobiol B 2002;67:32-8.

22. Bianchi B, Campolmi P, Mavilia L, Danesi A, Rossi R, Cappugi P. Monochromatic excimer light (308 nm): an immunohistochemical study of cutaneous $T$ cells and apoptosis-related molecules in psoriasis. J Eur Acad Dermatol Venereol 2003;17:408-13.

23. Cui J, Shen LY, Wang GC. Role of hair follicles in the repigmentation of vitiligo. J Invest Dermatol 1991;97:410-6.

24. Goldstein NB, Koster MI, Hoaglin LG, Spoelstra NS, Kechris KJ, Robinson SE, et al. Narrow band ultraviolet B treatment for human vitiligo is associated with proliferation, migration, and differentiation of melanocyte precursors. J Invest Dermatol 2015;135:2068-76.

25. Yang YS, Cho HR, Ryou JH, Lee MH. Clinical study of repigmentation patterns with either narrow-band ultraviolet $\mathrm{B}$ (NBUVB) or $308 \mathrm{~nm}$ excimer laser treatment in Korean vitiligo patients. Int J Dermatol 2010;49:317-23.

26. Lee CH, Wu SB, Hong CH, Yu HS, Wei YH. Molecular Mechanisms of UV-Induced Apoptosis and Its Effects on Skin Residential Cells: The Implication in UV-Based Phototherapy. Int J Mol Sci 2013;14:6414-35.

27. Imokawa G. Autocrine and paracrine regulation of melanocytes in human skin and in pigmentary disorders. Pigment Cell Res 2004; 17:96-110.

28. Noborio R, Morita A. Preferential induction of endothelin-1 in a human epidermal equivalent model by narrow-band ultraviolet B light sources. Photodermatol Photoimmunol Photomed 2010;26:159-61.

29. Lan CC, Yu HS, Lu JH, Wu CS, Lai HC. Irradiance, but not fluence, plays a crucial role in UVB-induced immature pigment cell development: new insights for efficient UVB phototherapy. Pigment Cell Melanoma Res 2013;26:367-76.
30. Yu HS, Wu CS, Yu CL, Kao YH, Chiou MH. Helium-neon laser irradiation stimulates migration and proliferation in melanocytes and induces repigmentation in segmental-type vitiligo. J Invest Dermatol 2003;120:56-64.

31. Byun JW, Babitha S, Kim EK, Shin J. A successful helium-neon laser and topical tacrolimus combination therapy in one child with vitiligo. Dermatol Ther 2015;28:333-5.

32. Wu CS, Hu SC, Lan CC, Chen GS, Chuo WH, Yu HS. Lowenergy helium-neon laser therapy induces repigmentation and improves the abnormalities of cutaneous microcirculation in segmental-type vitiligo lesions. Kaohsiung J Med Sci 2008;24:180-9.

33. Lan CC, Wu SB, Wu CS, Shen YC, Chiang TY, Wei YH, et al. Induction of primitive pigment cell differentiation by visible light (helium-neon laser): a photoacceptor-specific response not replicable by UVB irradiation. J Mol Med (Berl) 2012;90:321-30.

34. Savant SS. Therapeutic spot and regional dermabrasion in stable vitiligo. Indian J Dermatol Venereol Leprol 1996;62:13945.

35. Sethi S, Mahajan BB, Gupta RR, Ohri A. Comparative evaluation of the therapeutic efficacy of dermabrasion, dermabrasion combined with topical 5\% 5-fluorouracil cream, and dermabrasion combined with topical placentrex gel in localized stable vitiligo. Int J Dermatol 2007;46:875-9.

36. Bayoumi W, Fontas E, Sillard L, Le Duff F, Ortonne JP, Bahadoran $\mathrm{P}$, et al. Effect of a preceding laser dermabrasion on the outcome of combined therapy with narrowband ultraviolet $\mathrm{B}$ and potent topical steroids for treating nonsegmental vitiligo in resistant localizations. Br J Dermatol 2012;166:208-11.

37. Anbar T, Westerhof W, Abdel-Rahman A, El-Khayyat M, ElMetwally Y. Treatment of periungual vitiligo with erbium-YAGlaser plus 5-flurouracil: a left to right comparative study. J Cosmet Dermatol 2006;5:135-9.

38. Tierney EP, Kouba DJ, Hanke CW. Review of fractional photothermolysis: treatment indications and efficacy. Dermatol Surg 2009;35:1445-61.

39. Shin J, Lee JS, Hann SK, Oh SH. Combination treatment by 10 $600 \mathrm{~nm}$ ablative fractional carbon dioxide laser and narrowband ultraviolet $\mathrm{B}$ in refractory nonsegmental vitiligo: a prospective, randomized half-body comparative study. $\mathrm{Br} J$ Dermatol 2012;166:658-61.

40. Li L, Wu Y, Li L, Sun Y, Qiu L, Gao XH, et al. Triple combination treatment with fractional $\mathrm{CO} 2$ laser plus topical betamethasone solution and narrowband ultraviolet $\mathrm{B}$ for refractory vitiligo: a prospective, randomized half-body, comparative study. Dermatol Ther 2015;28:131-4.

41. Vachiramon V, Chaiyabutr C, Rattanaumpawan P, Kanokrungsee $\mathrm{S}$. Effects of a preceding fractional carbon dioxide laser on the outcome of combined local narrowband ultraviolet B and topical steroids in patients with vitiligo in 
difficult-to-treat areas. Lasers Surg Med 2016;48:197-202.

42. Li L, Fukunaga-Kalabis M, Yu H, Xu X, Kong J, Lee JT, et al. Human dermal stem cells differentiate into functional epidermal melanocytes. J Cell Sci 2010;123:853-60.

43. Bae JM, Eun SH, Lee H, Lee JH, Kim GM. Comparison of 311nm Ti:Sapphire laser vs. 308-nm Excimer laser treatment for vitiligo: A prospective randomized controlled non-inferiority trial. Vitiligo International Symposium; 2016 December 2-3; Rome, Italy.

44. Choi SY, Oh CT, Kwon TR, Kwon HJ, Choi EJ, Jang YJ, et al. Gain-switched 311-nm Ti:Sapphire laser might be a potential treatment modality for atopic dermatitis. Lasers Med Sci 2016;31:1437-45.

45. Zerbinati N, Portale S, Palmieri B, Rottigni V, lannitti T. A preliminary study to assess the efficacy of a new UVA1 laser for treatment of psoriasis. Photomed Laser Surg 2012;30:610-4.

46. Babino G, Giunta A, Esposito M, Saraceno R, Pavlidis A,

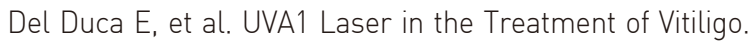
Photomed Laser Surg 2016;34:200-4.

47. Skov L, Hansen H, Allen M, Villadsen L, Norval M, Barker JN, et al. Contrasting effects of ultraviolet $A 1$ and ultraviolet $B$ exposure on the induction of tumour necrosis factor-alpha in human skin. Br J Dermatol 1998;138:216-20.

48. Lee SC, Bae JM, Choi GS. Quality of life in patients with vitiligo in Korea: A nationwide questionnaire-based study. 8th Annual Meeting of the ASPCR; 2016 November 12-13; Kaohsiung, Taiwan. 